\title{
Acyclovir-induced immune thrombocytopenia: Case report and review of the literature
}

\author{
GYULA LÁSZLÓ FEKETE ${ }^{1}$, LÁSZLÓ FEKETE ${ }^{1}$, ROBERT ANCUCEANU ${ }^{2}$, \\ SIMONA LAURA IANOȘI ${ }^{3}$, MIRUNA DRĂGĂNESCU ${ }^{4}$ and ILARIE BRIHAN ${ }^{5}$
}

\author{
${ }^{1}$ Department of Dermatology, Dermatology Clinic, 'George Emil Palade' University of Medicine, Pharmacy, \\ Science and Technology, 540139 Târgu Mureș; ${ }^{2}$ Department of Pharmaceutical Botany and Cell Biology, \\ Faculty of Pharmacy, 'Carol Davila' University of Medicine and Pharmacy, 020956 Bucharest; \\ ${ }^{3}$ Department of Dermatology, University of Medicine and Pharmacy, 200349 Craiova; \\ ${ }^{4}$ Medical Department, Faculty of Medicine and Pharmacy, University 'Dunărea de Jos', \\ 800010 Galati; ${ }^{5}$ Department of Dermatology, Dermatology Clinic, Faculty of Medicine \\ and Pharmacy, University of Oradea, 410073 Oradea, Romania
}

Received May 15, 2020; Accepted June 16, 2020

DOI: $10.3892 / \mathrm{etm} .2020 .8971$

\begin{abstract}
There are a number of medications which can serve as catalysts for drug-induced immune thrombocytopenia (DIPT). A minimum of six different mechanisms have been put forward as the means by which drug-induced antibodies can encourage platelet destruction, thus emphasising the complexity of the pathogenesis of DITP. Acyclovir, has been widely used because of its highly potent prohibitive properties for infections caused by HSV and VZV. The common adverse effects of this drug are well known, the severe adverse reactions are mostly related to high dose intravenous administrations. The immune thrombocytopenia induced by acyclovir is unusual. The authors present a rare clinical case of acyclovir-induced immune thrombocytopenia in a 72-year-old female patient with typical herpes zoster treated with acyclovir. The clinical and laboratory findings, taken together with the transitory relationship between acycolvir and the start of thrombocytopenia, combined with the elimination of the other know sources of thrombocytopenia, allowed us to reach the diagnosis of acyclovir-induced immune thrombocytopenia. An international database search
\end{abstract}

Correspondence to: Dr Simona Laura Ianoși, Department of Dermatology, University of Medicine and Pharmacy, 2 Petru Rareș Street, 200349 Craiova, Romania

E-mail:dermafek@yahoo.com

Dr László Fekete, Department of Dermatology, Dermatology Clinic, 'George Emil Palade' University of Medicine, Pharmacy, Science and Technology, 12 Gheorghe Doja Street, 540139 Târgu Mures,, Romania

E-mail: lacifek@yahoo.com

Key words: acyclovir, herpes zoster, thrombocytopenia, drug induced, adverse reactions was employed to complete an extensive review of the current literature. Contemporary information on acyclovir-induced immune thrombocytopenia was collected by the analysis of present day review articles and accessible case reports. The authors found five published cases of acyclovir-induced immune thrombocytopenia. Analyzing these articles it was concluded that immune thrombocytopenia induced by acyclovir is rare, and an unusual side effect, with good prognosis. Prompt diagnosis is vital to appropriate management, therefore clinicians need to be cognisant of this rare potential adverse reaction.

\section{Introduction}

There are a number of medications which can serve as catalysts for drug-induced immune thrombocytopenia (DIPT) (1-3). The majority of DITP cases result in mild symptoms, but there have been documented instances of dangerous bleeding. A minimum of six different mechanisms have been put forward as the means by which drug-induced antibodies can encourage platelet destruction, thus emphasising the complexity of the pathogenesis of DITP. It is technically possible to identify the antibodies that react with platelets when they come in contact with the sensitising drug, but due to the complexity of the testing and the rarity of the necessary equipment and expertise, this is often not practical. Rather, in the vast majority of cases the decision to halt the suspected medication of subjects who experience DITP is better made on clinical grounds (4). Acyclovir, an acyclic purine nucleoside analogue, has been widely used because of its highly potent prohibitive properties for infections caused by HSV and VZV (5). Nausea, diarrhea, dizziness, headaches, and mental changes are the most common and well-known side effects of this drug. Severe adverse effects such as neurotoxicity, kidney disorders and psychiatric effects are not common, mostly related to high dose intravenous administrations (6-8). Immune thrombocytopenia induced by acyclovir is unusual. 


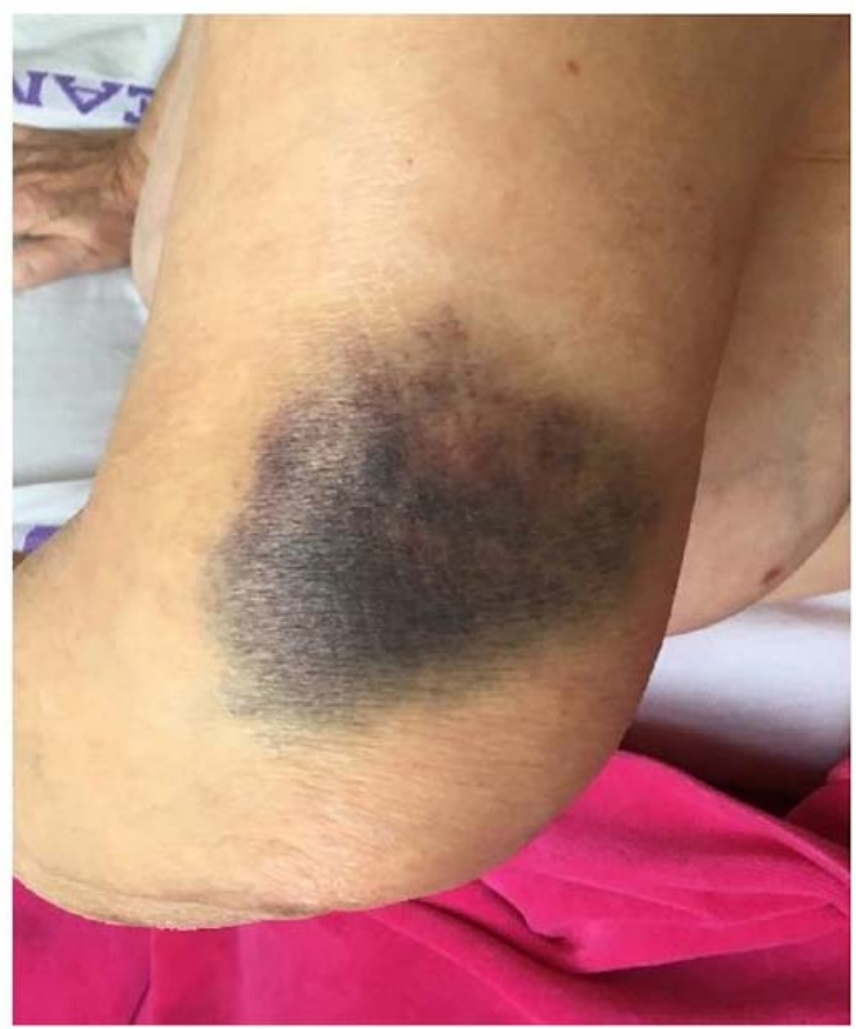

Figure 1. Clinical aspect. Hematoma on the left cubital area.

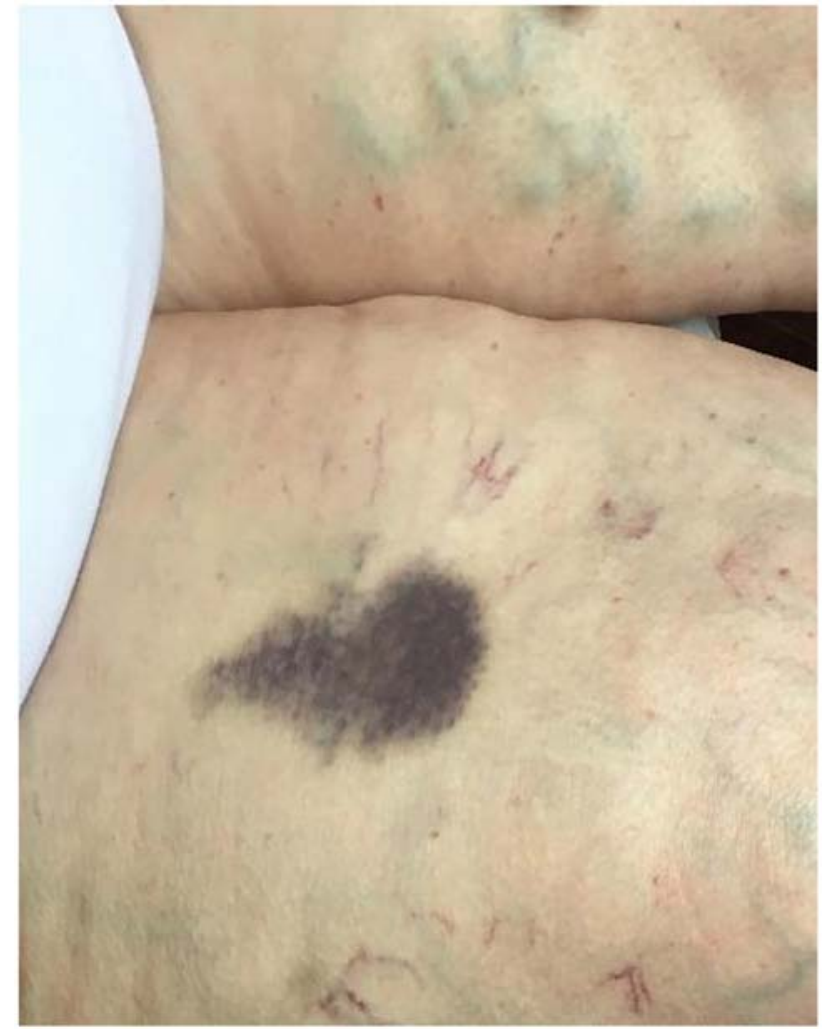

Figure 2. Clinical aspect. Hematoma on the right femoral area.

using enzyme-linked immunoassay (ELISA) which was positive. The clinical and laboratory findings, taken together with the transitory relationship between acyclovir and the start of thrombocytopenia, combined with the elimination of the other know sources of thrombocytopenia, allowed us to reach the diagnosis of acyclovir-induced immune thrombocytopenia. The administration of acyclovir was discontinued and oral prednisone treatment was introduced at $1 \mathrm{mg} / \mathrm{kg}$ body weight dose. The platelet count was checked every second day and began to rise 10 days after discontinuation of acyclovir and returned to normal values over a period of 15 days. After this period the dose of prednisone was decreased with $5 \mathrm{mg}$, every 3 days. The patient left the hospital clinically healed with normal levels of hematological laboratory findings including a normal platelet count level.

\section{Discussion}

Epidemiologic studies performed in the US and Europe suggest that $\sim 10$ persons per million are affected by DITP each year (9). The real incidence is unknown, but could be higher in elderly especially with associated severe co-morbidities and hospitalized persons, who are more likely to be exposed to different medications. The first report of DITP was published in 1865 (10). Despite the low frequency, DITP is important to recognize because $>200$ drugs, including some herbal medicines, that have been reported to be causative (11). A minimum of six different mechanisms have been put forward as the means by which drug-induced antibodies can encourage platelet destruction, thus emphasising the complexity of the pathogenesis of DITP. The majority of DITPs are believed to prove a drug-induced immune thrombocytopenia, detection of acyclovir-dependent platelet antibodies was performed 
have a drug-dependent immune mechanism as their source. How drugs induce platelet antibodies and how platelets are destroyed by these antibodies are still unclear (4). The clinical diagnosis of DITP is based on a few key features like: a) extensive petechiae, ecchymosis or hematomas appeared a few days after taking the putative drug, b) markedly low blood platelet levels appeared 3-10 days after starting a putative medication, c) platelet counts return to normal at $\sim 7$ days after stopping the putative drug (usually in 1-10 days) and d) exclusion of common known causes of thrombocytopenia $(12,13)$. In laboratory findings the presence of the specific drug-dependent platelet antibodies are crucial for the diagnosis. Also a careful, detailed personal and family history is important to patient evaluation. After examining methodologically and in detail the instances of drug-induced thrombocytopenia through 2008, George et al (14) constructed a database which catalogued the suspected drugs. The database is updated at regular intervals and can be accessed online at http://www.ouhsc.edu/platelets. They also devised a set of four clinical criteria and four levels of evidence to assess the likelihood that individual drugs are capable of causing DITP. In our case, the clinical aspect, the exclusion of other etiologies of thrombocytopenia, platelet count fluctuation, and the positive anti-platelet antibodies, and with the temporal relationship between the acyclovir and the start of thrombocytopenia allowed us to reach the diagnosis of acyclovir-induced immune thrombocytopenia. Based on the published clinical criteria we ranked the relationship between the drug and the disease on level 2 of evidence meeting three criteria of the four. The first step in the treatment is to discontinue medication in a patient with possible DITP. In many cases platelet levels start to increase without any medication. It is recommended as a common practice to administer corticosteroids, but whether they are beneficial in patients with DITP is not established. Other treatment options are mentioned in the literature i.e. platelet transfusion, intravenous $\mathrm{IgG}$, or even plasma exchange with different results (15-17). Our patient was treated with oral corticosteroid with a good result. An international database search was employed to complete an extensive review of the current literature. Contemporary information on acyclovir-induced immune thrombocytopenia was further informed by the analysis of present day review articles and accessible case reports. Search of PubMed/MEDLINE, Google Scholar, and Web of Science databases found five published cases of acyclovir-induced immune thrombocytopenia (18-22). Acyclovir treatment was indicated for herpes simplex infection in three cases $(18,20,22)$ and for zoster in two cases, similarly to our case $(19,21)$. For the herpes simplex infections the treatment was oral, like in our case, in the other cases the administration of the drug was parenteral. All the cases were males aged between 20 and 81 years, except our presented case. The evolution in all the cases was similar, the clinical findings and the lower level of platelet count appeared after 4-8 days after starting the acyclovir treatment. In all the cases the specific drug-dependent platelet antibodies were done, with positive results. In all cases the treatment was stopped and different treatment options were used. Oral prednisone in one case, as in our case, intravenous IgG in one case, platelet mass transfusion in one case and in the others supportive treatment was used. In two cases the patients were without remarkable co-morbidities, similarly to our presented case $(19,21)$. The other patients had severe co-morbidities such as systemic lupus, AIDS and chronic obstructive pulmonary disease, and were also treated for these diseases $(18,20,22)$. In every case the evolution was good, platelet count levels became normal in 10-15 days (18-22). The immune thrombocytopenia induced by acyclovir is rare and an unusual side effect of a commonly used drug. Most DITPs are thought to be caused by, still unclear, drug-dependent immune mechanism. In elderly patients, and especially those with associated severe co-morbidities, who need to be treated with acyclovir, regular platelet count measurement should be considered. Prompt diagnosis is vital to appropriate management, therefore clinicians need to be cognisant of this rare potential adverse reaction $(23,24)$.

\section{Acknowledgements}

Not applicable.

\section{Funding}

No funding was received.

\section{Availability of data and materials}

All data generated or analyzed during this study are included in this published article.

\section{Authors' contributions}

GLF was responsible for the clinical management of the patient, the evaluation and analysis of the data, and the writing of the manuscript. LF was involved in the conception of the study and was responsible for the preparation and revisions of the manuscript. RA, SLI, and MD were responsible for the literature research and the revisions of the manuscript. IB was involved in the conception of the study and contributed to the writing of the manuscript. The final version of the article was read and approved by all the authors.

\section{Ethics approval and consent to participate}

The study was approved by the Ethics Committee of the Romanian College of Physicians of Mures County (Târgu Mureș, Romania).

\section{Patient consent for publication}

Written informed consent was obtained from the patient.

\section{Competing interests}

The authors declare that they have no competing interests.

\section{Authors' information}

GLF: Associate Professor of Dermatology, Department of Dermatology, Dermatology Clinic, 'George Emil Palade' University of Medicine, Pharmacy, Science and Technology (Târgu Mureş, Romania). 


\section{References}

1. Tatu AL, Elisei AM, Chioncel V, Miulescu M and Nwabudike LC: Immunologic adverse reactions of $\beta$-blockers and the skin (Review). Exp Ther Med 18: 955-959, 2019.

2. Fekete GL and Fekete L: Cutaneous leukocytoclastic vasculitis associated with erlotinib treatment: A case report and review of the literature. Exp Ther Med 17: 1128-1131, 2019.

3. Tatu AL, Ciobotaru OR, Miulescu M, Buzia OD, Elisei AM, Mardare N, Diaconu C, Robu S and Nwabudike LC: Hydrochlorothiazide: Chemical structure, therapeutic, phototoxic and carcinogenetic effects in dermatology. Rev Chim (Bucharest) 69: 2110-2114, 2018.

4. Aster RH, Curtis BR, McFarland JG and Bougie DW: Drug-induced immune thrombocytopenia: Pathogenesis, diagnosis, and management. J Thromb Haemost 7: 911-918, 2009.

5. Dworkin RH, Johnson RW, Breuer J, Gnann JW, Levin MJ, Backonja M, Betts RF, Gershon AA, Haanpaa ML, McKendrick MW, et al: Recommendations for the management of herpes zoster. Clin Infect Dis 44 (Suppl 1): S1-S26, 2007.

6. Busso M and Berman B: Antivirals in dermatology. J Am Acad Dermatol 32: 1031-1040, 1995.

7. Adair JC, Gold M and Bond RE: Acyclovir neurotoxicity: Clinical experience and review of the literature. South Med J 87: $1227-1231,1994$

8. Haefeli WE, Schoenenberger RA, Weiss P and Ritz RF: Acyclovir-induced neurotoxicity: Concentration - side effect relationship in acyclovir overdose. Am J Med 94: 212-215, 1993.

9. van den Bemt PM, Meyboom RH and Egberts AC: Drug-induced immune thrombocytopenia. Drug Saf 27: 1243-1252, 2004.

10. Vipan W: Quinine as a cause of purpura. Lancet 86: 37, 1865.

11. Aster RH and Bougie DW: Drug-induced immune thrombocytopenia. N Engl J Med 357: 580-587, 2007.

12. Chong BH: Drug-induced immune thrombocytopenia. Platelets 2: 173-181, 1991.

13. Visentin GP and Liu CY: Drug-induced thrombocytopenia. Hematol Oncol Clin North Am 21: 685-696, vi, 2007.
14. George JN, Raskob GE, Shah SR, Rizvi MA, Hamilton SA, Osborne $S$ and Vondracek T: Drug-induced thrombocytopenia: A systematic review of published case reports. Ann Intern Med 129: 886-890, 1998.

15. Crosby WH: Editorial: Wet purpura, dry purpura. JAMA 232: 744-745, 1975.

16. Ray JB, Brereton WF and Nullet FR: Intravenous immune globulin for the treatment of presumed quinidine-induced thrombocytopenia. DICP 24: 693-695, 1990.

17. Pourrat O: Treatment of drug-related diseases by plasma exchanges. Ann Med Interne (Paris) 145: 357-360, 1994.

18. Tsappa I, Missouris C and Psarellis S: Acyclovir-induced thrombocytopenia in a patient with SLE. BMJ Case Rep 2018: pii: bcr-2018-225118, 2018.

19. Hong X, Wang X and Wang Z: A rare case report of acyclovirinduced immune thrombocytopenia with tongue hematomas as the first sign, and a literature review. BMC Pharmacol Toxicol 18: $12,2017$.

20. Kamboj J, Wu F, Kamboj R, Suzue K and Khosla P: A rare case of acyclovir-induced thrombocytopenia. Am J Ther 21: e159-162, 2014.

21. Katsenos S, Gkolias D and Nikolopoulou M: Acyclovir-induced immune thrombocytopenia in a patient with herpes zoster of the trigeminal nerve. Pharmacotherapy 30: 1085-1086, 2010.

22. Cersosimo RJ, Hankins MA and Hamrah SD: An unusual case of thrombocytopenia and leukopenia associated with diltiazem and acyclovir. J Pharm Technol 19: 154-158, 2003.

23. Fekete GL, Cotoi OS and Fekete JE: Multiple nodular cutaneous metastasis as first clinical sign of signet ring cell gastric carcinoma - case report. Acta Dermatovenerol Croat 20: 34-37, 2012.

24. Brănişteanu DE, Pintilie A, Andreş LE, Dimitriu A, Oanţă A, Stoleriu G and Brănişteanu DC: Ethiopatogenic hypotheses in lichen planus. Rev Med Chir Soc Med Nat Iasi 120: 760-767, 2016. 